\title{
Navier-Stokes equations with variable viscosity in variable exponent spaces of Clifford-valued functions
}

\author{
Rui Niu ${ }^{1,2}$, Hongtao Zheng ${ }^{1}$ and Binlin Zhang ${ }^{2 *}$
}

"Correspondence:
zhangbinlin2012@163.com
2Department of Mathematics,
Heilongjiang Institute of
Technology, Harbin, 150050, China
Full list of author information is
available at the end of the article

available at the end of the article

\begin{abstract}
In this paper we study the stationary generalized Navier-Stokes equations when the viscosity is not only a constant but also a function which depends on the position and the shear-velocity. For this we establish an improved decomposition of variable exponent Lebesgue spaces of Clifford-valued functions. Using this decomposition together with Clifford operator calculus, we obtain the existence, uniqueness and representation of solutions for the generalized Stokes equations and the generalized Navier-Stokes equations with variable viscosity in the setting of variable exponent spaces of Clifford-valued functions. Furthermore, the equivalences of solutions and weak solutions for the aforementioned equations are justified.
\end{abstract}

MSC: 30G35; 35J60; 35Q30; 46E30; 76D03

Keywords: Clifford analysis; variable exponent; variable viscosity; Stokes equations; Navier-Stokes equations

\section{Introduction}

In this paper we are concerned with the stationary generalized Navier-Stokes equations:

$$
\begin{aligned}
& -\operatorname{div}(A \nabla u)+\rho(u \cdot \nabla) u+\nabla q=\rho f \quad \text { in } \Omega, \\
& \operatorname{div} u=0 \quad \text { in } \Omega, \\
& u=0 \quad \text { on } \partial \Omega,
\end{aligned}
$$

where the operator $A$ is defined by $A u=a u$ with $a(x, u): \bar{\Omega} \times \mathbb{R}^{n} \rightarrow \mathbb{R}^{+}$and $a \in C^{\infty}(\bar{\Omega} \times$ $\left.\mathbb{R}^{n}\right)$ and $\Omega \subset \mathbb{R}^{n}(n \geq 2)$ is a bounded domain with sufficiently smooth boundary $\partial \Omega, u$ is the velocity, $q$ the hydrostatic pressure, $\rho$ the density, $f$ the vector of the external forces. Notice that (1.1) may be written

$$
-\frac{1}{\rho} \operatorname{div}(A(x, u) \mathrm{D} u)+(u \cdot \nabla) u+\nabla \pi=f \quad \text { in } \Omega,
$$

where $\pi:=q / \rho$. If the relation $A(x, u):=\mu\left(\alpha+|\mathrm{D} u|^{2}\right)^{\frac{p-2}{2}}$ with $\mu, \alpha>0$, where $\mu$ is the shear viscosity, $\mathrm{D} u:=(1 / 2)\left(\nabla u+(\nabla u)^{\top}\right)$ is the symmetric gradient, then the fluid is called nonNewtonian fluid with p-structure; see, for example, Acerbi and Mingione [1] for related

\section{Springer}

(c) 2015 Niu et al.; licensee Springer. This is an Open Access article distributed under the terms of the Creative Commons Attribution License (http://creativecommons.org/licenses/by/4.0), which permits unrestricted use, distribution, and reproduction in any medium, provided the original work is properly credited. 
discussions in this direction. Clearly, the fluid in the case $p=2$ is a Newtonian fluid, and then (1.4) becomes

$$
-v \Delta u+(u \cdot \nabla) u+\nabla \pi=f \quad \text { in } \Omega,
$$

where $v:=\mu / \rho$ is the kinematic viscosity. Here (1.5) is the famous Navier-Stokes equation, for the detailed account about the Navier-Stokes equations we refer to [2, 3]. In this paper we will consider the Navier-Stokes equations in a Clifford language under the assumption that the viscosity depends on the position and velocity, i.e., $v=v(x, u) \in \mathbb{R}^{+}$. It is easy to see that the viscous term $-v \Delta u$ can be replaced by the Clifford expression $D v D u$, where $D=\sum_{j=1}^{n} \mathrm{e}_{j} \partial_{j}$ denotes the Dirac operator of a massless field, $u$ is a Clifford-valued function; for the details as regards the Clifford algebra we refer to the next section.

In order to study the time-dependent motion of a viscous, incompressible fluid we need velocity fields $u$ of the particles of the fluid along with their first spatial derivatives to be summable to the $p(k)$ th power $\left(p_{-} \geq 1, k \in \mathbb{N}\right)$ at each time and each position. Additionally, $u$ has to be solenoidal and vanishes at the boundary of the domain where the motion happens. Thus this gives rise to the introduction of spaces of divergence free functions in a generalized sense. Furthermore, the models of electrorheological fluids, which were introduced by Rajagopal and Růžička $[4,5]$, can be described by the boundary value problems for the generalized Navier-Stokes equations. This leads naturally to the establishment of function spaces with variable exponents. On the other hand, Clifford algebras have important applications in a variety of fields including geometry, theoretical physics and digital image processing. They are named after the English geometer William Kingdon Clifford. We have a generalization of the complex numbers, the quaternions, and the exterior algebras; see [6]. As an active branch of mathematics over the past 40 years, Clifford analysis usually studies the solutions of the Dirac equation for functions defined on domains in Euclidean space and taking value in Clifford algebras; see, for example, [7]. In [8] the authors gave an overview of applications of Clifford analysis in mathematical physics. Hence, it makes sense to study the stationary Navier-Stokes equations in the setting of Clifford algebras.

It is worth pointing out that our attempt is to give a unified approach to deal with physical problems modeled by the generalized Navier-Stokes equations, which is quite different from the approaches of some authors, for example, we refer the reader to the monograph [9]. Based on the above consideration, we should study the generalized Navier-Stokes equations in variable exponent spaces of Clifford-valued functions. Of course, the study of variable exponent spaces has been driven by various problems in elastic mechanics, calculus of variations and differential equations with variable growth; see [10-20] and the references therein.

Evidently, we should primarily be concerned with the study of the first term $D A(D u)$ in (1.1). In [21, 22], Nolder first introduced the general nonlinear $A$-Dirac equations $D A(x, D u)=0$ which arise in the study of many phenomena in the physical sciences. In particular, he developed some tools for the study of weak solutions to nonlinear $A$-Dirac equations in the space $W_{0}^{1, p}\left(\Omega, C \ell_{n}\right)$. Inspired by his works, Fu and Zhang in [23, 24] were interested in the existence of weak solutions for the general nonlinear $A$-Dirac equations with variable growth. For this purpose, the authors established a theory of variable exponent spaces of Clifford-valued functions with applications to homogeneous and non- 
homogeneous $A$-Dirac equations; see also [25, 26]. Very recently, Fu et al. in [27, 28] established a Hodge-type decomposition of variable exponent Lebesgue spaces of Cliffordvalued functions with applications to the Stokes equations, the Navier-Stokes equations and the $A$-Dirac equations $D A(D u)=0$. By using the Hodge-type decomposition and variational methods, Molica Bisci et al. in [29] studied the properties of weak solutions to the homogeneous and nonhomogeneous $A$-Dirac equations with variable growth. For an overview for the nonlinear $A$-Dirac equations with variable growth, we refer the reader to [30].

It is worth mentioning that Diening et al. in [31] studied the following model introduced in $[4,5]$ to describe the motions of electrorheological fluids:

$$
\begin{cases}-\operatorname{div} \mathcal{M}(\mathrm{D} u)+(u \cdot \nabla) u+\nabla \pi=f, & x \in \Omega, \\ \operatorname{div} u=0, & x \in \Omega, \\ u=0, & x \in \partial \Omega,\end{cases}
$$

where $f \in\left(W_{0}^{1, p(x)}(\Omega)\right)^{*}=W^{-1, p^{\prime}(x)}(\Omega), 2 n /(n+2)<p_{-} \leq p_{+}<\infty$ and the operator $A$ satisfies certain natural variable growth conditions. The authors obtained the existence of weak solutions in $\left(W_{0}^{1, p(x)}(\Omega)\right)^{n} \times L_{0}^{s}(\Omega)$, here $s:=\min \left\{\left(p_{+}\right)^{\prime}, n p_{-} / 2\left(n-p_{-}\right)\right\}$if $p_{-}<n$ and $s:=\left(p_{+}\right)^{\prime}$ otherwise, $L_{0}^{s}(\Omega):=\left\{\pi \in L^{s}(\Omega): \int_{\Omega} \pi d x=0\right\}$. From a practical point of view, we have to investigate the representation of solutions to system (1.6) besides existence and uniqueness. Based on the method developed by Sprößig in [2], it is possible to obtain the desired results if we consider system (1.6) as $\mathcal{M}(\mathrm{D} u)=a(x, u) \mathrm{D} u$ under the assumption $f \in W^{-1, p(x)}\left(\Omega, \mathbb{R}^{n}\right)$, here $a(x, u)$ is a positive function.

Motivated by their works, our goal in this article is to give a generalization of related results in Sprößig [2] to the variable exponent setting. More precisely, we investigate properties of solutions for the following Navier-Stokes equations in variable exponent spaces of Clifford-valued functions:

$$
\begin{cases}D A D u+[u D]_{0} u+D \pi=f, & x \in \Omega, \\ {[D u]_{0}=0,} & x \in \Omega, \\ u=0, & x \in \partial \Omega .\end{cases}
$$

Throughout the paper, the operator $A$ is defined by $A u=$ au with $a(x, u): \bar{\Omega} \times \mathbb{R}^{n} \rightarrow \mathbb{R}^{+}$and $a \in C^{\infty}\left(\bar{\Omega} \times \mathbb{R}^{n}\right)$. The outline for this study is to first establish a modified decomposition of variable exponent Lebesgue spaces. Then the classic results about the Stokes equations and the Navier-Stokes equations obtained by Gürlebeck and Sprößig [32, 33] are extended to the variable exponent setting. In particular, we would like to point out the equivalences of solutions and weak solutions for the above-mentioned equations, which has not been clearly stated by the previous works in [28].

This paper is organized as follows. In Section 2, we start with a brief summary of Clifford algebra and variable exponent spaces of Clifford-valued functions. In Section 3, we establish a modified decomposition of variable exponent Lebesgue spaces, and then discuss its some applications, which will be needed later. In Section 4, we obtain the existence and uniqueness of the generalized Stokes equations in the context of variable exponent spaces. Moreover, the equivalence of solutions and weak solutions for the abovementioned equations are showed. In Section 5, using similar methods to [28], we prove 
the existence and uniqueness of solutions to the generalized Navier-Stokes equations in $W_{0}^{1, p(x)}\left(\Omega, C \ell_{n}\right) \times L^{p(x)}(\Omega, \mathbb{R})$ under certain hypotheses. Moreover, the equivalence of solutions and weak solutions for the above-mentioned equations are presented.

\section{Preliminaries}

First we recall some related definitions and results concerning Clifford algebra and variable exponent spaces of Clifford-valued functions. For a detailed account we refer to [6, 34, 35]. Let $C \ell_{n}$ be the real universal Clifford algebra over $\mathbb{R}^{n}$. Denote $C \ell_{n}$ by

$$
\mathrm{C} \ell_{n}=\operatorname{span}\left\{\mathrm{e}_{0}, \mathrm{e}_{1}, \mathrm{e}_{2}, \ldots, \mathrm{e}_{n}, \mathrm{e}_{1} \mathrm{e}_{2}, \ldots, \mathrm{e}_{n-1} \mathrm{e}_{n}, \ldots, \mathrm{e}_{1} \mathrm{e}_{2} \cdots \mathrm{e}_{n}\right\}
$$

where $\mathrm{e}_{0}=1$ (the identity element in $\mathbb{R}^{n}$ ), $\left\{\mathrm{e}_{1}, \mathrm{e}_{2}, \ldots, \mathrm{e}_{n}\right\}$ is an orthonormal basis of $\mathbb{R}^{n}$ with the relation $\mathrm{e}_{i} \mathrm{e}_{j}+\mathrm{e}_{j} \mathrm{e}_{i}=-2 \delta_{i j} \mathrm{e}_{0}$. Thus the dimension of $C \ell_{n}$ is $2^{n}$. For $I=\left\{i_{1}, \ldots, i_{r}\right\} \subset$ $\{1, \ldots, n\}$ with $1 \leq i_{1}<i_{2}<\cdots<i_{n} \leq n$, put $\mathrm{e}_{I}=\mathrm{e}_{i_{1}} \mathrm{e}_{i_{2}} \cdots \mathrm{e}_{i_{r}}$, while for $I=\emptyset, \mathrm{e}_{\emptyset}=\mathrm{e}_{0}$. For $0 \leq r \leq n$ fixed, the space $\mathrm{C} \ell_{n}^{r}$ is defined by

$$
\mathrm{C} \ell_{n}^{r}=\operatorname{span}\left\{\mathrm{e}_{I}:|I|=r\right\}
$$

where $|I|$ denotes cardinal number of the set $I$. The Clifford algebra $C \ell_{n}$ is a graded algebra as

$$
\mathrm{C} \ell_{n}=\bigoplus_{r} \mathrm{C} \ell_{n}^{r}
$$

Any element $a \in \mathrm{C} \ell_{n}$ may thus be written in a unique way as

$$
a=[a]_{0}+[a]_{1}+\cdots+[a]_{n}
$$

where []$_{r}: \mathrm{C} \ell_{n} \rightarrow \mathrm{C} \ell_{n}^{r}$ denotes the projection of $\mathrm{C} \ell_{n}$ onto $\mathrm{C} \ell_{n}^{r}$. In particular, by $\mathrm{C} \ell_{n}^{2}=\mathbb{H}$ we denote the algebra of real quaternions. It is customary to identify $\mathbb{R}$ with $C \ell_{n}^{0}$ and identify $\mathbb{R}^{n}$ with $C \ell_{n}^{1}$, respectively. This means that each element $x$ of $\mathbb{R}^{n}$ may be represented by

$$
x=\sum_{i=1}^{n} x_{i} \mathrm{e}_{i} .
$$

For $u \in \mathrm{C} \ell_{n}$, we denote by $[u]_{0}$ the scalar part of $u$, that is, the coefficient of the element $\mathrm{e}_{0}$. We define the Clifford conjugation as follows:

$$
\overline{\mathrm{e}_{i_{1}} \mathrm{e}_{i_{2}} \cdots \mathrm{e}_{i_{r}}}=(-1)^{\frac{r(r+1)}{2}} \mathrm{e}_{i_{1}} \mathrm{e}_{i_{2}} \cdots \mathrm{e}_{i_{r}} .
$$

We denote

$$
(A, B)=[\bar{A} B]_{0}
$$

Then an inner product is thus obtained, giving rise to the norm $|\cdot|$ on $C \ell_{n}$ given by

$$
|A|^{2}=[\bar{A} A]_{0} .
$$


A Clifford-valued function $u: \Omega \rightarrow C \ell_{n}$ can be written as $u=\sum_{I} u_{I} \mathrm{e}_{I}$, where the coefficients $u_{I}: \Omega \rightarrow \mathbb{R}$ are real-valued functions.

The Dirac operator on Euclidean space used here is introduced by

$$
D=\sum_{j=1}^{n} \mathrm{e}_{j} \partial_{j}
$$

This is a special case of the Atiyah-Singer-Dirac operator acting on sections of a spinor bundle. Note that the most famous Dirac operator describes the propagation of a free fermion in three dimensions.

If $u$ is a real-valued function defined on a domain $\Omega$ in $\mathbb{R}^{n}$, then $D u=\nabla u=\left(\partial_{1} u, \partial_{2} u\right.$, $\left.\ldots, \partial_{n} u\right)$. Moreover, $D^{2}=D D=-\Delta$, where $\Delta$ is the Laplace operator which operates only on coefficients. A function is left monogenic if it satisfies the equation $D u(x)=0$ for each $x \in \Omega$. A similar definition can be given for right monogenic function. An important example of a left monogenic function is the generalized Cauchy kernel

$$
G(x)=\frac{1}{\omega_{n}} \frac{\bar{x}}{|x|^{n}},
$$

where $\omega_{n}$ denotes the surface area of the unit ball in $\mathbb{R}^{n}$. This function is a fundamental solution of the Dirac operator.

Next we recall some basic properties of variable exponent spaces of Clifford-valued functions. Note that in what follows, we use the short notation $L^{p(x)}(\Omega), W^{1, p(x)}(\Omega)$, etc., instead of $L^{p(x)}(\Omega, \mathbb{R}), W^{1, p(x)}(\Omega, \mathbb{R})$, etc. Throughout this paper we always assume (unless declare specially)

$$
p \in \mathcal{P}^{\log }(\Omega) \quad \text { and } \quad 1<p_{-}:=\inf _{x \in \bar{\Omega}} p(x) \leq p(x) \leq \sup _{x \in \bar{\Omega}} p(x)=: p_{+}<\infty
$$

where $\mathcal{P}^{\log }(\Omega)$ is the set of exponent $p$ satisfying the so-called log-Hölder continuity, i.e.,

$$
|p(x)-p(y)| \leq \frac{C}{\log \left(\mathrm{e}+|x-y|^{-1}\right)}
$$

holds for all $x, y \in \Omega$; see [36]. Let $\mathcal{P}(\Omega)$ be the set of all Lebesgue measurable functions $p: \Omega \rightarrow(1, \infty)$. Given $p \in \mathcal{P}(\Omega)$ we define the conjugate function $p^{\prime}(x) \in \mathcal{P}(\Omega)$ by

$$
p^{\prime}(x)=\frac{p(x)}{p(x)-1}, \quad \forall x \in \Omega
$$

The variable exponent Lebesgue space $L^{p(x)}(\Omega)$ is defined by

$$
L^{p(x)}(\Omega)=\left\{u \in \mathcal{P}(\Omega): \int_{\Omega}|u(x)|^{p(x)} d x<\infty\right\}
$$

with the norm

$$
\|u\|_{L^{p(x)}(\Omega)}=\inf \left\{t>0: \int_{\Omega}\left|\frac{u(x)}{t}\right|^{p(x)} d x \leq 1\right\}
$$


The variable exponent Sobolev space $W^{1, p(x)}(\Omega)$ is defined by

$$
W^{1, p(x)}(\Omega)=\left\{u \in L^{p(x)}(\Omega):|\nabla u| \in L^{p(x)}(\Omega)\right\}
$$

with the norm

$$
\|u\|_{W^{1, p(x)}(\Omega)}=\|\nabla u\|_{L^{p(x)}(\Omega)}+\|u\|_{L^{p(x)}(\Omega)} .
$$

Denote $W_{0}^{1, p(x)}(\Omega)$ by the completion of $C_{0}^{\infty}(\Omega)$ in $W^{1, p(x)}(\Omega)$ with respect to the norm (2.2). The space $W^{-1, p(x)}(\Omega)$ is defined as the dual of the space $W_{0}^{1, p^{\prime}(x)}(\Omega)$. For more details we refer to [9] and references therein.

In what follows, we say that $u \in L^{p(x)}\left(\Omega, C \ell_{n}\right)$ can be understood coordinate wisely. For example, $u \in L^{p(x)}\left(\Omega, C \ell_{n}\right)$ means that $\left\{u_{I}\right\} \subset L^{p(x)}(\Omega)$ for $u=\sum_{I} u_{I} e_{I} \in \mathrm{C} \ell_{n}$ with the norm $\|u\|_{L^{p(x)}\left(\Omega, \mathrm{C} \ell_{n}\right)}=\sum_{I}\left\|u_{I}\right\|_{L^{p(x)}(\Omega)}$. In this way, spaces $W^{1, p(x)}\left(\Omega, C \ell_{n}\right), W_{0}^{1, p(x)}\left(\Omega, C \ell_{n}\right)$, $C_{0}^{\infty}\left(\Omega, C \ell_{n}\right)$, etc. can be understood similarly. In particular, the space $L^{2}\left(\Omega, C \ell_{n}\right)$ can be converted into a right Hilbert $\mathrm{C} \ell_{n}$-module by defining the following Clifford-valued inner product (see [32, Definition 3.74]):

$$
(f, g)_{\mathrm{C} \ell_{n}}=\int_{\Omega} \overline{f(x)} g(x) d x .
$$

Remark 2.1 A simple calculation leads to the claim that the norm $\|u\|_{L^{p(x)}\left(\Omega, \mathrm{C} \ell_{n}\right)}$ is equivalent to the norm $\||u|\|_{L^{p(x)}(\Omega)}$. Furthermore, $\|D u\|_{L^{p(x)}\left(\Omega, C \ell_{n}\right)}$ is an equivalent norm of $\|u\|_{W_{0}^{1, p(x)}\left(\Omega, C \ell_{n}\right)}$ for every $u \in W_{0}^{1, p(x)}\left(\Omega, C \ell_{n}\right)$; for the details we refer to [24, 25].

Lemma 2.1 (see $[23,24])$ If $p(x) \in \mathcal{P}(\Omega)$, then $L^{p(x)}\left(\Omega, C \ell_{n}\right)$ and $W^{1, p(x)}\left(\Omega, C \ell_{n}\right)$ are reflexive Banach spaces.

Definition 2.1 (see [32]) Let $u \in C\left(\Omega, C \ell_{n}\right)$. The Teodorescu operator is defined by

$$
T u(x)=\int_{\Omega} G(x-y) u(y) d y,
$$

where $G(x)$ is the generalized Cauchy kernel mentioned above.

Lemma 2.2 (see [28]) The following operators are bounded linear operators:

(i) $T: L^{p(x)}\left(\Omega, C \ell_{n}\right) \rightarrow W^{1, p(x)}\left(\Omega, C \ell_{n}\right)$.

(ii) $\widetilde{T}: W^{-1, p(x)}\left(\Omega, C \ell_{n}\right) \rightarrow L^{p(x)}\left(\Omega, C \ell_{n}\right)$, where the operator $\widetilde{T}$ can be considered as a unique bounded linear extension of the Teodorescu operator.

Lemma 2.3 (see [28]) The following operators are bounded linear operators:

(i) $D: W^{1, p(x)}\left(\Omega, C \ell_{n}\right) \rightarrow L^{p(x)}\left(\Omega, C \ell_{n}\right)$.

(ii) $\widetilde{D}: L^{p(x)}\left(\Omega, C \ell_{n}\right) \rightarrow W^{-1, p(x)}\left(\Omega, C \ell_{n}\right)$, where the operator $\widetilde{D}$ can be considered as a unique bounded linear extension of the Dirac operator.

Lemma 2.4 (see [28]) Let $p(x) \in \mathcal{P}(\Omega)$.

(i) If $u \in W_{0}^{1, p(x)}\left(\Omega, C \ell_{n}\right)$, then the equation $T D u(x)=u(x)$ holds for all $x \in \Omega$.

(ii) If $u \in L^{p(x)}\left(\Omega, C \ell_{n}\right)$, then the equation $D T u(x)=u(x)$ holds for all $x \in \Omega$. 
Lemma 2.5 (see [28]) Let $p(x)$ satisfies (2.1).

(i) If $u \in L^{p(x)}\left(\Omega, C \ell_{n}\right)$, then the equation $\widetilde{T} \widetilde{D} u(x)=u(x)$ holds for all $x \in \Omega$.

(ii) If $u \in W^{-1, p(x)}\left(\Omega, C \ell_{n}\right)$, then the equation $\widetilde{D} \widetilde{T} u(x)=u(x)$ holds for all $x \in \Omega$.

\section{A modified decomposition of spaces}

Gürlebeck and Sprößig [32,33] showed that the orthogonal decomposition of the space $L^{2}(\Omega)$ holds in the hyper-complex function theory:

$$
L^{2}\left(\Omega, C \ell_{n}\right)=\left(\operatorname{ker} D \cap L^{2}\left(\Omega, C \ell_{n}\right)\right) \oplus D W_{0}^{1,2}\left(\Omega, C \ell_{n}\right)
$$

with respect to the Clifford-valued product (2.3). Kähler [37] extended the orthogonal decomposition (3.1) to the spaces $L^{p}\left(\Omega, C \ell_{n}\right)$ in form of a direct decomposition in a bounded domain. In [38], Cerejeiras and Kähler investigated a direct decomposition of $L^{p}\left(\Omega, C \ell_{n}\right)$ in an unbounded domain. In [28], Zhang et al. generalized (3.1) to the setting of $L^{p(x)}\left(\Omega, C \ell_{n}\right)$ in a bounded domain.

In [36], Diening et al. showed that the Dirichlet problem of the Poisson equation with homogeneous boundary data

$$
\begin{cases}-\Delta u=f & \text { in } \Omega, \\ u=0 & \text { on } \partial \Omega,\end{cases}
$$

has a unique weak solution $u \in W^{1, p(x)}(\Omega)$ for each $f \in W^{-1, p(x)}(\Omega)$. Moreover, the following estimate holds:

$$
\|u\|_{W^{1, p(x)}(\Omega)} \leq C(n, p, \Omega)\|f\|_{W^{-1, p(x)}(\Omega)} .
$$

Here $u$ is called a weak solution of problem (3.2) provided that

$$
\langle f, \varphi\rangle=\int_{\Omega} \nabla u \cdot \nabla \varphi d x, \quad \forall \varphi \in W_{0}^{1, p^{\prime}(x)}(\Omega) .
$$

Then it is easy to see that for all $f \in W^{-1, p(x)}\left(\Omega, C \ell_{n}\right)$ the problem (3.2) still has a unique weak solution $u \in W^{1, p(x)}\left(\Omega, C \ell_{n}\right)$. We denote by $\Delta_{0}^{-1}$ the solution operator.

Now we are in a position to prove a decomposition of the variable exponent Lebesgue spaces equipped with the modified inner product.

Theorem 3.1 The space $L^{p(x)}\left(\Omega, C \ell_{n}\right)$ allows the Hodge-type decomposition

$$
L^{p(x)}\left(\Omega, C \ell_{n}\right)=\left(B \operatorname{ker} \widetilde{D} \cap L^{p(x)}\left(\Omega, C \ell_{n}\right)\right) \oplus D W_{0}^{1, p(x)}\left(\Omega, C \ell_{n}\right)
$$

with respect to the inner product

$$
(f, g)_{A}=\int_{\Omega} \overline{A f(x)} A g(x) d x,
$$

where $A B=B A=I$ and $B u:=b(x, u) u$ with $a b=1$. 
Proof Similar to the proof of Theorem 6 in [37], we first show the intersection of spaces $B \operatorname{ker} \widetilde{D} \cap L^{p(x)}\left(\Omega, C \ell_{n}\right)$ and spaces $D W_{0}^{1, p(x)}\left(\Omega, C \ell_{n}\right)$ contains one and only one zero element. Indeed, suppose $f \in\left(B \operatorname{ker} \widetilde{D} \cap L^{p(x)}\left(\Omega, C \ell_{n}\right)\right) \cap D W_{0}^{1, p(x)}\left(\Omega, C \ell_{n}\right)$, then $\widetilde{D} A f=0$. Moreover, $f \in L^{p(x)}\left(\Omega, \mathrm{C} \ell_{n}\right)$ shows that there exists a function $w=T A f \in W^{1, p(x)}\left(\Omega, \mathrm{C} \ell_{n}\right)$ because of Lemma 2.2; then $f=B D w \in D W_{0}^{1, p(x)}\left(\Omega, C \ell_{n}\right)$ due to Lemma 2.4. It follows that $w \in W_{0}^{1, p(x)}\left(\Omega, C \ell_{n}\right)$. Therefore, we have $\widetilde{D} D w=0$ in $\Omega$ and $w=0$ on $\partial \Omega$. From the uniqueness of $\Delta_{0}^{-1}$ we obtain $w=0$. Consequently, $f=0$. Therefore, the sum of the two subspaces is a direct one.

Now let $u \in L^{p(x)}\left(\Omega, C \ell_{n}\right)$. If we take $u_{2}=B D \Delta_{0}^{-1} \widetilde{D} A u$, then using the same arguments as the first part, we deduce that $u_{2} \in D W_{0}^{1, p(x)}\left(\Omega, C \ell_{n}\right)$. Let $u_{1}=u-u_{2}$. Then $u_{1} \in$ $L^{p(x)}\left(\Omega, C \ell_{n}\right)$. Furthermore, we take $u_{k} \in W_{0}^{1, p(x)}\left(\Omega, C \ell_{n}\right)$ such that $u_{k} \rightarrow u$ in $L^{p(x)}\left(\Omega, C \ell_{n}\right)$, then by the density of $W_{0}^{1, p(x)}\left(\Omega, C \ell_{n}\right)$ in $L^{p(x)}\left(\Omega, C \ell_{n}\right)$ and Lemma 2.3 , we have for any $\varphi \in W_{0}^{1, p^{\prime}(x)}\left(\Omega, C \ell_{n}\right)$,

$$
\left(u_{1}, B D \varphi\right)_{A}=\int_{\Omega} \overline{A\left(u-u_{2}\right)} D \varphi d x=\lim _{k \rightarrow \infty} \int_{\Omega} \overline{\left(D A u_{k}-D A B D \Delta_{0}^{-1} D A u_{k}\right)} \varphi d x=0 .
$$

Thus, we obtain $u_{1} \in B \operatorname{ker} \widetilde{D}$. Since $u \in L^{p(x)}\left(\Omega, C \ell_{n}\right)$ is arbitrary, the desired result follows.

From this decomposition we can get the following projections:

$$
\begin{aligned}
& P_{a}: L^{p(x)}\left(\Omega, C \ell_{n}\right) \rightarrow B \operatorname{ker} \widetilde{D} \cap L^{p(x)}\left(\Omega, C \ell_{n}\right), \\
& Q_{a}: L^{p(x)}\left(\Omega, C \ell_{n}\right) \rightarrow D W_{0}^{1, p(x)}\left(\Omega, C \ell_{n}\right) .
\end{aligned}
$$

Moreover, we have

$$
Q_{a}=B D \Delta_{0}^{-1} \widetilde{D} A, \quad P_{a}=I-Q_{a}
$$

Corollary 3.1 Let $f \in L^{p(x)}\left(\Omega, C \ell_{n}\right)$. Then the following equations with homogeneous boundary data:

$$
\begin{cases}D A D u=f & \text { in } \Omega \\ u=0 & \text { on } \partial \Omega\end{cases}
$$

has a unique solution $u \in W_{0}^{2, p(x)}\left(\Omega, C \ell_{n}\right)$ which may be represented by the formula $u=$ $T Q_{a} B T f$.

Proof For existence of solution, Theorem 3.1 implies that there exists a function $u \in$ $W_{0}^{1, p(x)}\left(\Omega, C \ell_{n}\right)$ such that $Q_{a} B T f=D u$. Lemma 2.2 gives $T f \in W^{1, p(x)}\left(\Omega, C \ell_{n}\right)$ and $Q_{a} B T f \in$ $W_{0}^{2, p(x)}\left(\Omega, C \ell_{n}\right)$. Then from Lemma 2.4 it follows that $T Q_{a} B T f=T D u=u$. Hence $u \in$ $W_{0}^{2, p(x)}\left(\Omega, C \ell_{n}\right)$. Further, we have

$$
D A D u=D A Q_{a} B T f=D A B T f-D A P_{a} B f=f,
$$

which implies $D A D u=f$. 
For the uniqueness of solution, consider the boundary value problems: $D A D u=0$ in $\Omega$ and $u=0$ on $\partial \Omega$. It is easy to see that $D u \in \operatorname{im} P_{a} \cap \operatorname{im} Q_{a}=\{0\}$. Then $D u=0$. Hence $u=T D u=0$ because of Lemma 2.4 .

Remark 3.1 Gürlebeck and Sprößig [33] pointed out that the equations of linear elasticity and time-independent Maxwell equations over a three-dimensional domain with zero boundary condition could be transformed into problem (3.5) in a quaternionic language; see Section 4.3 and Section 4.4 in [33] for more details.

Corollary 3.2 The space $L^{p(x)}\left(\Omega, C \ell_{n}\right) \cap \operatorname{im} Q_{a}$ is a closed subspace of $L^{p(x)}\left(\Omega, C \ell_{n}\right)$.

Proof The proof follows that of Lemma 6 in [28] almost word by word, we thus omit the details.

Corollary 3.3 $\left(L^{p(x)}\left(\Omega, C \ell_{n}\right) \cap \operatorname{im} Q_{a}\right)^{*}=L^{p^{\prime}(x)}\left(\Omega, C \ell_{n}\right) \cap \operatorname{im} Q_{a}$. In other words, the linear operator

$$
\Phi: D W_{0}^{1, p^{\prime}(x)}\left(\Omega, C \ell_{n}\right) \rightarrow\left(D W_{0}^{1, p(x)}\left(\Omega, C \ell_{n}\right)\right)^{*}
$$

given by

$$
\Phi(D u)(D \varphi)=[D \varphi, D u]_{A}:=\int_{\Omega}[\overline{A D \varphi} A D u]_{0} d x
$$

is a Banach space isomorphism.

Proof Similar to the proof of Lemma 2.7 in [28], for the reader's convenience, we will give a detailed treatment of the proof. In view of Corollary 3.2, $D W_{0}^{1, p(x)}\left(\Omega, C \ell_{n}\right)$ and $D W_{0}^{1, p^{\prime}(x)}\left(\Omega, C \ell_{n}\right)$ are reflexive Banach spaces since they are closed in $L^{p(x)}\left(\Omega, C \ell_{n}\right)$ and $L^{p^{\prime}(x)}\left(\Omega, C \ell_{n}\right)$, respectively. Linearity of $\Phi$ is clear. For injectivity, suppose

$$
\Phi(D u)(D \varphi)=[D \varphi, D u]_{A}=0
$$

for all $\varphi \in W_{0}^{1, p(x)}\left(\Omega, C \ell_{n}\right)$ and some $u \in W_{0}^{1, p^{\prime}(x)}\left(\Omega, C \ell_{n}\right)$. For any $\omega \in L^{p(x)}\left(\Omega, C \ell_{n}\right)$, according to (3.4), we may write $\omega=\alpha+\beta$ with $\alpha \in B \operatorname{ker} \widetilde{D} \cap L^{p(x)}\left(\Omega, C \ell_{n}\right)$ and $\beta \in$ $D W_{0}^{1, p(x)}\left(\Omega, C \ell_{n}\right)$. Thus we obtain

$$
[\omega, D u]_{A}=[\alpha+\beta, D u]_{A}=[\alpha, D u]_{A}+[\beta, D u]_{A}
$$

Taking a similar argument to the first part of the proof in Theorem 3.1, we may write $\alpha=B \zeta$ with $\zeta \in \operatorname{ker} \widetilde{D} \cap L^{p(x)}\left(\Omega, C \ell_{n}\right)$ and $D u=B D \eta$ with $\eta \in W_{0}^{1, p(x)}\left(\Omega, C \ell_{n}\right)$, then it is easy to see that $(\alpha, D u)_{\mathrm{Sc}}=0$. This together with (3.6) gives $(\omega, D u)_{\mathrm{Sc}}=0$. This leads to $D u=0$. It follows that $\Phi$ is injective. To get surjectivity, let $f \in\left(D W_{0}^{1, p(x)}\left(\Omega, C \ell_{n}\right)\right)^{*}$. By the Hahn-Banach theorem, there is $F \in\left(L^{p(x)}\left(\Omega, C \ell_{n}\right)\right)^{*}$ with $\|F\|=\|f\|$ and $\left.F\right|_{D W_{0}^{1, p(x)}\left(\Omega, C \ell_{n}\right)}=f$. Moreover, there exists $\varphi \in L^{p^{\prime}(x)}\left(\Omega, \mathrm{C} \ell_{n}\right)$ such that $F(u)=(u, \varphi)_{\mathrm{Sc}}$ for any $u \in L^{p(x)}\left(\Omega, \mathrm{C} \ell_{n}\right)$. According to (3.4), we can write $\varphi=\xi+D \alpha$, where $\xi \in B \operatorname{ker} \widetilde{D} \cap L^{p^{\prime}(x)}\left(\Omega, C \ell_{n}\right), D \alpha \in D W_{0}^{1, p^{\prime}(x)}\left(\Omega, C \ell_{n}\right)$. For any $D u \in D W_{0}^{1, p(x)}\left(\Omega, C \ell_{n}\right)$, we have

$$
f(D u)=[D u, \varphi]_{A}=[D u, \xi]_{A}+[D u, D \alpha]_{A}=[D u, D \alpha]_{A}=\Phi(D \alpha)(D u)
$$


Consequently, $\Phi(D \alpha)=f$. It follows that $\Phi$ is surjective. By Theorem 3.1 in [23] we have

$$
|\Phi(D u)(D \varphi)|=\left|[D \varphi, D u]_{A}\right| \leq C\|D \varphi\|_{L^{p(x)}\left(\Omega, \mathrm{C} \ell_{n}\right)}\|D u\|_{L^{p^{\prime}(x)}\left(\Omega, \mathrm{C} \ell_{n}\right)} .
$$

This means that $\Phi$ is continuous. Furthermore, it is immediate that $\Phi^{-1}$ is continuous from the inverse function theorem. The proof of Corollary 3.3 is thus finished.

\section{Stokes equations with variable viscosity}

In the study of the stationary Navier-Stokes equations, the corresponding Stokes equations play an important role. It can be said that any open question about the Navier-Stokes equations, such as global existence of strong solutions, uniqueness and regularity of weak solutions, and asymptotic behavior, is closely related with the qualitative and quantitative properties of the solutions of Stokes equations; see, for example, [38] for related discussions. To be precise, it is crucial to investigate the properties of solutions $(u, \pi)$ to the following Stokes system:

$$
\begin{aligned}
& -v \Delta u+\nabla \pi=f \quad \text { in } \Omega, \\
& \operatorname{div} u=f_{0} \quad \text { in } \Omega, \\
& u=v_{0} \quad \text { on } \partial \Omega .
\end{aligned}
$$

With $\int_{\Omega} f_{0} d x=\int_{\partial \Omega} n \cdot v_{0} d x$, the necessary condition for the solvability is given. The scalar function $f_{0}$ is a measure of the compressibility of fluid. The boundary condition (4.3) describes the adhesion at the boundary of the domain $\Omega$ for $v_{0}=0$. This system describes the stationary flow of a homogeneous viscous fluid for small Reynold's numbers. For more details we refer to $[3,33]$.

For $f=\sum_{i=1}^{n} f_{i} \mathrm{e}_{i}$ and $u=\sum_{i=1}^{n} u_{i} \mathrm{e}_{i}$, let us consider the following Stokes system in the hyper-complex formulation:

$$
\begin{aligned}
& \widetilde{D} A D u+D \pi=f \quad \text { in } \Omega, \\
& {[D u]_{0}=0 \quad \text { in } \Omega,} \\
& u=0 \quad \text { on } \partial \Omega .
\end{aligned}
$$

Definition 4.1 We say that $(u, \pi) \in W_{0}^{1, p(x)}\left(\Omega, C \ell_{n}\right) \times L^{p(x)}(\Omega)$ a solution of (4.4)-(4.6) provided that it satisfies the system (4.4)-(4.6) for every $f \in W^{-1, p(x)}\left(\Omega, C \ell_{n}\right)$.

Definition 4.2 The operator $\widetilde{\nabla}: L^{p(x)}(\Omega) \rightarrow\left(W^{-1, p(x)}(\Omega)\right)^{n}$ is defined by

$$
\langle\widetilde{\nabla} f, \varphi\rangle=-\langle f, \operatorname{div} \varphi\rangle:=-\int_{\Omega} f \operatorname{div} \varphi d x
$$

for all $f \in L^{p(x)}(\Omega)$ and $\varphi \in\left(C_{0}^{\infty}(\Omega)\right)^{n}$.

Lemma 4.1 (see [28]) Let $\Omega$ be a bounded Lipschitz domain of $\mathbb{R}^{n}$. Let $f \in\left(W^{-1, p(x)}(\Omega)\right)^{n}$ satisfy

$$
\langle f, \varphi\rangle=\int_{\Omega} f \cdot \varphi d x=0
$$


for any $\varphi \in \mathscr{M}(\Omega):=\left\{v \in\left(W_{0}^{1, p^{\prime}(x)}(\Omega)\right)^{n}: \operatorname{div} v=0\right\}$. Then there exists $q \in L^{p(x)}(\Omega)$ such that $f=\widetilde{\nabla} q$.

Theorem 4.1 Let $f \in W^{-1, p(x)}\left(\Omega, C \ell_{n}\right)$. Then the Stokes system (4.4)-(4.6) has a unique solution $(u, \pi) \in W_{0}^{1, p(x)}\left(\Omega, C \ell_{n}\right) \times L^{p(x)}(\Omega)$ in the form

$$
u+T Q_{a} B \pi=T Q_{a} B \widetilde{T} f
$$

with respect to the estimate

$$
\|D u\|_{L^{p(x)}\left(\Omega, C \ell_{n}\right)}+\left\|Q_{a} B \pi\right\|_{L^{p(x)}(\Omega)} \leq C\left\|Q_{a} B \widetilde{T} f\right\|_{L^{p(x)}\left(\Omega, C \ell_{n}\right)} .
$$

Here, $C \geq 1$ is a constant and the hydrostatic pressure $\pi$ is unique up to a constant.

Proof We first show that the following representation is valid for each $f \in W^{-1, p(x)}\left(\Omega, C \ell_{n}\right)$ :

$$
T Q_{a} B \widetilde{T} f=u+T Q_{a} B \pi
$$

Indeed, let $\varphi_{n} \in W_{0}^{1, p(x)}\left(\Omega, C \ell_{n}\right)$ with $\varphi_{n} \rightarrow \varphi$ in $L^{p(x)}\left(\Omega, C \ell_{n}\right)$. By Lemma 2.4, we have

$$
\operatorname{TQBT}\left(D \varphi_{n}\right)=T Q_{a} B \varphi_{n}
$$

Since $W_{0}^{1, p(x)}\left(\Omega, C \ell_{n}\right)$ is dense in $L^{p(x)}\left(\Omega, C \ell_{n}\right)$, it follows that $T Q_{a} B \widetilde{T} \widetilde{D} \varphi=T Q_{a} B \varphi$. Thus, for $u \in W_{0}^{1, p(x)}\left(\Omega, C \ell_{n}\right)$ and $\pi \in L^{p(x)}(\Omega)$, Lemma 2.4 yields

$$
T Q_{a} B \widetilde{T} f=T Q_{a} B \widetilde{T}(\widetilde{D} A D u+\widetilde{D} \pi)=u+T Q_{a} B \pi .
$$

This implies that our system (4.4)-(4.6) is equivalent to the system

$$
\begin{aligned}
& u+T Q_{a} B \pi=T Q_{a} B \widetilde{T} f \\
& {\left[Q_{a} B \pi\right]_{0}=\left[Q_{a} B \widetilde{T} f\right]_{0} .}
\end{aligned}
$$

Clearly, the equality (4.7) is equivalent to the following equality:

$$
D u+Q_{a} B \pi=Q_{a} B \widetilde{T} f .
$$

Now we need to show that for each $f \in W^{-1, p(x)}\left(\Omega, C \ell_{n}^{1}\right)$, the function $Q_{a} B T f$ can be decomposed into two functions $D u$ and $Q_{a} B \pi$. Suppose $D u+Q_{a} B \pi=0$ for $u \in$ $W_{0}^{1, p(x)}\left(\Omega, C \ell_{n}^{1}\right) \cap$ kerdiv and $\pi \in L^{p(x)}(\Omega)$. Then (4.5) gives $\left[Q_{a} B \pi\right]_{0}=0$. Thus, $Q_{a} B \pi=0$. Hence, $D u=Q_{a} B \pi=0$. This means that $D u+Q_{a} B \pi$ is a direct sum, which is a subset of $\operatorname{im} Q_{a}$.

Next we have to consider the existence of a functional $\mathcal{F} \in\left(L^{p(x)}\left(\Omega, C \ell_{n}^{1}\right) \cap \mathrm{im} Q_{a}\right)^{*}$ with $\mathcal{F}(D u)=0$ and $\mathcal{F}\left(Q_{a} B \pi\right)=0$ but $\mathcal{F}\left(Q_{a} B \widetilde{T} f\right) \neq 0$. This is equivalent to asking if there exists $g \in W^{-1, p^{\prime}(x)}\left(\Omega, C \ell_{n}^{1}\right)$, such that for all $u \in W_{0}^{1, p(x)}\left(\Omega, C \ell_{n}^{1}\right) \cap \operatorname{ker} \operatorname{div}$ and $\pi \in L^{p(x)}(\Omega)$,

$$
\left[D u, Q_{a} B \widetilde{T} g\right]_{A}=0,
$$




$$
\left[Q_{a} B \pi, Q_{a} B \widetilde{T} g\right]_{A}=0,
$$

but $\left[Q_{a} B \widetilde{T} f, Q_{a} B \widetilde{T} g\right]_{A} \neq 0$. Here, Corollary 3.3 is applied.

Thus, let us consider the system (4.10) and (4.11) with $g \in W^{-1, p^{\prime}(x)}\left(\Omega, C \ell_{n}^{1}\right)$. Taking the arguments as the first part of the proof in Theorem 3.1, we may write $D u=B D w$ with $w \in W_{0}^{1, p(x)}\left(\Omega, C \ell_{n}^{1}\right)$. Then $\operatorname{div} u=\operatorname{div} w=0$ because of $b(x)>0$. According to Lemma 2.5, (4.10) yields

$$
\begin{aligned}
0 & =\left[D u, Q_{a} B \widetilde{T} g\right]_{A}=\int_{\Omega} \overline{A D u} A Q_{a} B \widetilde{T} g d x \\
& =\int_{\Omega} \bar{w} \widetilde{D} A Q_{a} B \widetilde{T} g d x=\int_{\Omega} \bar{w} g d x-\int_{\Omega} \bar{w} \widetilde{D} A P_{a} B \widetilde{T} g d x=\int_{\Omega} \bar{w} g d x,
\end{aligned}
$$

which implies $g=\widetilde{\nabla} h=\widetilde{D} h$ with $h \in L^{p^{\prime}(x)}(\Omega)$ because of Lemma 4.1. Thus we obtain from (4.11) and Lemma 2.4

$$
\left[Q_{a} B \pi, Q_{a} B \widetilde{T} g\right]_{A}=\left[Q_{a} B \pi, Q_{a} B \widetilde{T} \widetilde{D} h\right]_{A}=\left[Q_{a} B \pi, Q_{a} B h\right]_{A}=0
$$

holds for each $\pi \in L^{p(x)}(\Omega)$. Hence, $Q_{a} B \pi=\left|Q_{a} B h\right|^{p^{\prime}(x)-2} Q_{a} B h$ gives $Q_{a} B h=0$. Then we obtain

$$
g=\widetilde{D} h=\widetilde{D} A Q_{a} B h+\widetilde{D} A P_{a} B h=0
$$

Furthermore, we get

$$
\left[Q_{a} B \widetilde{T} f, Q_{a} B \widetilde{T} g\right]_{A}=0, \quad \forall f \in W^{-1, p(x)}\left(\Omega, C \ell_{n}^{1}\right)
$$

Finally, (4.9) yields

$$
\|D u\|_{L^{p(x)}\left(\Omega, C \ell_{n}\right)}+\left\|Q_{a} B \pi\right\|_{L^{p(x)}(\Omega)} \geq\left\|Q_{a} B \widetilde{T} f\right\|_{L^{p(x)}\left(\Omega, C \ell_{n}\right)} .
$$

By the norm equivalence theorem, we obtain

$$
\|D u\|_{L^{p(x)}\left(\Omega, C \ell_{n}\right)}+\left\|Q_{a} B \pi\right\|_{L^{p(x)}(\Omega)} \leq C\left\|Q_{a} B \widetilde{T} f\right\|_{L^{p(x)}\left(\Omega, C \ell_{n}\right)} .
$$

By Remark 2.1, Lemma 2.2, and the boundedness of the operator $Q_{a}$, we get

$$
\|u\|_{W_{0}^{1, p(x)}\left(\Omega, C \ell_{n}\right)}+\left\|Q_{a} B \pi\right\|_{L^{p(x)}(\Omega)} \leq C\|f\|_{W^{-1, p(x)}\left(\Omega, C \ell_{n}\right)} .
$$

From (4.12) the uniqueness of the solution follows. Note that $Q_{a} B\left(\pi_{1}-\pi_{2}\right)=0$ implies $B\left(\pi_{1}-\pi_{2}\right) \in B \operatorname{ker} \widetilde{D}$. Then $\pi_{1}=\pi_{2}+c$ with $c \in \mathbb{R}$. Therefore, $\pi$ is unique up to a constant. The proof of Theorem 4.1 is now complete.

In [28], the authors did not point out the relation of solutions and weak solutions for the Stokes equations. Next we should prove that solutions and weak solutions for the Stokes equations are equivalent. 
Definition 4.3 The Clifford-valued function $u$ is called a weak solution of the system (4.3)-(4.6), if for each $v \in W_{0}^{1, p^{\prime}(x)}\left(\Omega, C \ell_{n}^{1}\right) \cap$ ker div, the equality

$$
(A D u, D v)_{\mathrm{Sc}}=(f, v)_{\mathrm{Sc}}
$$

holds, where $(u, v)_{\mathrm{Sc}}$ denotes the classical scalar product $(u, v)_{\mathrm{Sc}}=\int_{\Omega}[\bar{u} v]_{0} d x=\int_{\Omega} \sum_{i=1}^{n} u_{i} \times$ $v_{i} d x$.

Theorem 4.2 Let $u \in W_{0}^{1, p(x)}\left(\Omega, C \ell_{n}^{1}\right)$ and $\pi \in L^{p(x)}(\Omega)$ be a solution of system (4.7)-(4.8). Then $u$ is a weak solution of system (4.4)-(4.6). If $u$ is a weak solution of the system (4.4)(4.6), then there exists a function $\pi \in L^{p(x)}(\Omega)$ such that the pair $(u, \pi)$ solves the system (4.7)-(4.8).

Proof We follow the idea of Theorem 4.5.2 in [33]. First we need to show that for all functions $v \in W_{0}^{1, p^{\prime}(x)}\left(\Omega, C \ell_{n}^{1}\right) \cap$ ker div, the following equality holds:

$$
\sum_{i=1}^{n}\left(A \nabla u_{i}, \nabla v_{i}\right)_{\mathrm{Sc}}=(f, v)_{\mathrm{Sc}}
$$

For this purpose, from (4.7) it follows that

$$
(A D u, D v)_{\mathrm{Sc}}=\left(A D T Q_{a} B \widetilde{T} f, D v\right)_{\mathrm{Sc}}-\left(A D T Q_{a} B \pi, D v\right)_{\mathrm{Sc}}
$$

for each $v \in W_{0}^{1, p^{\prime}(x)}\left(\Omega, C \ell_{n}^{1}\right) \cap$ ker div. The first term of the right side in (4.14) yields

$$
\left(A D T Q_{a} B \widetilde{T} f, D v\right)_{S_{c}}=\left(\widetilde{D} A D T Q_{a} B \widetilde{T} f, v\right)_{\mathrm{Sc}}=(f, v)_{\mathrm{Sc}},
$$

thanks to Lemma 2.4, Lemma 2.5 and (3.4). For the second term of the right side in (4.14), we choose a sequence $\left\{\pi_{k}\right\}_{k=1}^{\infty} \subset W_{0}^{1, p(x)}(\Omega)$ with $\pi_{k} \rightarrow \pi$ in $L^{p(x)}(\Omega)$, then

$$
\begin{aligned}
\sum_{i=1}^{n}\left(\left(A D T Q_{a} B \pi\right)_{i}, D v_{i}\right)_{\mathrm{Sc}} & =\lim _{k \rightarrow \infty} \sum_{i=1}^{n}\left(\left(A D T Q_{a} B \pi_{k}\right)_{i}, D v_{i}\right)_{\mathrm{Sc}} \\
& =\lim _{k \rightarrow \infty}\left(D A D T Q_{a} B \pi_{k}, v\right)_{\mathrm{Sc}}=\lim _{k \rightarrow \infty}\left(D \pi_{k}, v\right)_{\mathrm{Sc}}=0
\end{aligned}
$$

because $\left(\nabla \pi_{k}, v\right)_{\mathrm{Sc}}=-\left(\pi_{k}, \operatorname{div} v\right)_{\mathrm{Sc}}=0$ for all $v \in W_{0}^{1, p^{\prime}(x)}\left(\Omega, C \ell_{n}^{1}\right) \cap$ kerdiv. Then (4.14), together with (4.15) and (4.16), gives (4.13).

Conversely, let $u \in W_{0}^{1, p(x)}\left(\Omega, C \ell_{n}^{1}\right) \cap$ ker div be a weak solution for the system (4.4)-(4.6). That means that (4.13) holds. It easily follows from Lemma 2.4 and Theorem 3.1 that

$$
(A D u, D v)_{\mathrm{Sc}}-\left(A D T Q_{a} B \widetilde{T} f, D v\right)_{\mathrm{Sc}}+\left(A D T Q_{a} B q, D v\right)_{\mathrm{Sc}}=0
$$

for all $q \in L^{p(x)}(\Omega)$ and $v \in W_{0}^{1, p^{\prime}(x)}\left(\Omega, C \ell_{n}^{1}\right) \cap$ ker div. Then we have

$$
\sum_{i=1}^{n}\left(\nabla\left(u-T Q_{a} B \widetilde{T} f+T Q_{a} B q\right)_{i}, A \nabla v_{i}\right)_{\mathrm{Sc}}=0
$$


On one hand, we have shown in Theorem 4.1, which is independent of the above considerations, that the system

$$
\begin{aligned}
& \psi+T Q_{a} B \pi=T Q_{a} B \widetilde{T} f \\
& {\left[Q_{a} B \pi\right]_{0}=\left[Q_{a} B \widetilde{T} f\right]_{0}}
\end{aligned}
$$

has a solution $(\psi, \pi) \in W_{0}^{1, p(x)}\left(\Omega, C \ell_{n}^{1}\right) \times L^{p(x)}(\Omega)$. Then substituting $q$ in (4.17) by $\pi$, we obtain

$$
\left[D u-Q_{a} B \widetilde{T} f+Q_{a} B \pi\right]_{0}=0
$$

which implies

$$
u-T Q_{a} B \widetilde{T} f+T Q_{a} B \pi \in W_{0}^{1, p(x)}\left(\Omega, C \ell_{n}^{1}\right) \cap \text { ker div } .
$$

On the other hand, it follows from (4.17) that

$$
\left(D A D\left(u-T Q_{a} B \widetilde{T} f+T Q_{a} B \pi\right), v\right)_{\mathrm{Sc}}=0
$$

for all $v \in W_{0}^{1, p^{\prime}(x)}\left(\Omega, C \ell_{n}^{1}\right) \cap$ ker div. Obviously, $W_{0}^{1, p^{\prime}(x)}\left(\Omega, C \ell_{n}^{1}\right) \cap$ ker div is a closed subspace in $W_{0}^{1, p^{\prime}(x)}\left(\Omega, C \ell_{n}^{1}\right)$, and hence $W_{0}^{1, p^{\prime}(x)}\left(\Omega, C \ell_{n}^{1}\right) \cap$ ker div is a reflexive Banach space due to Lemma 2.1. Then (4.18) yields

$$
D A D\left(u-T Q_{a} B \widetilde{T} f+T Q_{a} B \pi\right)=0 .
$$

Finally, by using the density argument and Corollary 3.1 we obtain

$$
u-T Q_{a} B \widetilde{T} f+T Q_{a} B \pi=0 .
$$

This ends the proof of Theorem 4.2.

Remark 4.1 Actually, the last part of the proof in Theorem 4.2 is quite different from that of Theorem 4.5.2 in [33]. The reason lies in the difference of $W_{0}^{1,2}\left(\Omega, C \ell_{n}\right)$ and $W_{0}^{1, p(x)}\left(\Omega, C \ell_{n}\right)$.

\section{Navier-Stokes equations with variable viscosity}

Our aim in this section is to investigate the existence of solution of the time-independent generalized Navier-Stokes equations (1.7). Evidently, the main difference from the abovementioned Stokes equations is the appearance of the nonlinear convection term $(u \cdot \nabla) u$. In 1928, Oseen showed that one can get relatively good results if the convection term $(u \cdot \nabla) u$ is replaced by $(v \cdot \nabla) u$, where $v$ is a solution of the corresponding Stokes equations. In 1965, Finn [39] proved the existence of solutions for small external forces with a spatial decreasing to infinity of order $|x|^{-1}$ for the case of $n=3$ and used the Banach fixed-pointed theorem. Gürlebeck and Sprößig [32,33] solved this system by a reduction to a sequence of Stokes problems provided the external force $f$ belongs to $L^{p}(\Omega, \mathbb{H})$ for a bounded domain $\Omega$ and $6 / 5<p<3 / 2$. Cerejeiras and Kähler [38] obtained similar results 
provided the external force $f$ belongs to $W^{-1, p}\left(\Omega, C \ell_{n}\right)$ for an unbounded domain $\Omega$ and $n / 2 \leq p<\infty$. Zhang et al. [28] investigated similar results provided that the external force $f$ belongs to $W^{-1, p(x)}\left(\Omega, C \ell_{n}\right)$ for a bounded domain $\Omega$ and $n / 2 \leq p<\infty$. Our intention in this section is to extend these results to the more general case in variable exponent spaces.

For $f=\sum_{i=1}^{n} f_{i} \mathrm{e}_{i}, u=\sum_{i=1}^{n} u_{i} \mathrm{e}_{i}$, let us consider the following steady generalized NavierStokes equations in the hyper-complex notation:

$$
\begin{aligned}
& \widetilde{D} A D u+D \pi=F(u) \text { in } \Omega, \\
& {[D u]_{0}=0 \quad \text { in } \Omega,} \\
& u=0 \quad \text { on } \partial \Omega,
\end{aligned}
$$

with the nonlinear part $F(u)=f-[u D]_{0} u$, where the operator $A$ is mentioned above.

Lemma 5.1 (see [28]) Let $p(x)$ satisfies (2.1) and $n / 2 \leq p_{-} \leq p(x) \leq p_{+}<\infty$. Then the operator $F: W_{0}^{1, p(x)}\left(\Omega, C \ell_{n}^{1}\right) \rightarrow W^{-1, p(x)}\left(\Omega, C \ell_{n}^{1}\right)$ is a continuous operator and we have

$$
\left\|[u D]_{0} u\right\|_{W^{-1, p(x)}\left(\Omega, \mathrm{C} \ell_{n}\right)} \leq C_{1}\|u\|_{W_{0}^{1, p(x)}\left(\Omega, C \ell_{n}\right)^{\prime}}^{2}
$$

where $C_{1}=C_{1}(n, p, \Omega)$ is a positive constant.

Using Lemma 5.1, which is crucial to the convergence of the iteration method, we are able to give the main result as follows.

Theorem 5.1 Let $p(x)$ satisfy (2.1) and $n / 2 \leq p_{-} \leq p(x) \leq p_{+}<\infty$. Then the system (5.1)(5.3) has a unique solution $(u, \pi) \in W_{0}^{1, p(x)}\left(\Omega, C \ell_{n}\right) \times L^{p(x)}(\Omega, \mathbb{R})$ ( $\pi$ is unique up to a real constant) if the right-hand side $f$ satisfies the condition

$$
\|f\|_{W^{-1, p(x)}\left(\Omega, \mathrm{C} \ell_{n}\right)}<\frac{v^{2}}{4 C_{1} C_{4}^{2}},
$$

with $v=\mu / \rho, C_{4}=C_{2}\left(1+C_{3}\right)$, where $C_{3} \geq 1$ indicated in (5.8) and

$$
C_{2}=\|T\|_{\left[L^{p(x)} \cap \operatorname{im} Q, W_{0}^{1, p(x)}\right]}\|Q B\|_{\left[L^{\left.p(x), L^{p(x)} \cap \operatorname{im} Q\right]}\right.}\|\widetilde{T}\|_{\left[W^{\left.-1, p(x), L^{p(x)} \cap \operatorname{im} Q\right]}\right.} .
$$

For any function $u_{0} \in W_{0}^{1, p(x)}\left(\Omega, C \ell_{n}\right)$ with

$$
\left\|u_{0}\right\|_{W_{0}^{1, p(x)}\left(\Omega, C e_{n}\right)} \leq \frac{v}{2 C_{1} C_{2}}-\mathcal{F},
$$

here $\mathcal{F}=\sqrt{\frac{v^{2}}{4 C_{1}^{2} C_{4}^{2}}-\frac{1}{C_{1}}\|f\|_{W^{-1, p(x)}\left(\Omega, C C_{n}\right)}}$, the iteration procedure

$$
\begin{aligned}
& u_{k}+T Q B_{k-1} \pi_{k}=T Q B_{k-1} \widetilde{T} F\left(u_{k-1}\right), \quad k=1,2, \ldots \\
& {\left[Q B_{k-1} \pi_{k}\right]_{0}=\left[Q B_{k-1} \widetilde{T} F\left(u_{k-1}\right)\right]_{0}}
\end{aligned}
$$

converges in $W_{0}^{1, p(x)}\left(\Omega, C \ell_{n}\right) \times L^{p(x)}(\Omega)$, where $B_{k} h:=b\left(x, u_{k}\right) h$. 
Proof The proof is similar to that of Theorem 4.6.8 in [33]. For the reader's convenience, we will give the key details of the proof. Replacing $f$ by $F\left(u_{k-1}\right)$ in the proof of Theorem 4.1, we obtain the unique solvability of the Stokes equations (4.4)-(4.6) which we have to solve in each step. Moreover, we have the following estimate:

$$
\left\|D u_{k}\right\|_{L^{p(x)}\left(\Omega, C \ell_{n}\right)}+\left\|Q B \pi_{k}\right\|_{L^{p(x)}(\Omega)} \leq C_{3}\left\|Q B \widetilde{T} F\left(u_{k-1}\right)\right\|_{L^{p(x)}\left(\Omega, C \ell_{n}\right)},
$$

where $C_{3} \geq 1$ is a constant. In the following, using the Banach fixed point theorem, we could finish the remaining proof by following the proof of Theorem 3.1 in [28] word by word; we thus omit the details.

Definition 5.1 The Clifford-valued function $u$ is called a weak solution of the system (5.6)-(5.7), if for all $v \in W_{0}^{1, p(x)}\left(\Omega, C \ell_{n}^{1}\right) \cap \operatorname{ker} \operatorname{div}$, the following equality holds:

$$
(A D u, D v)_{\mathrm{Sc}}=(F(u), v)_{\mathrm{Sc}}
$$

that is to say, the following equality is valid:

$$
\sum_{i=1}^{n}\left(A \nabla u_{i}, \nabla v_{i}\right)_{\mathrm{Sc}}+\sum_{i=1}^{n}\left(u_{i} \partial_{i} u, v\right)_{\mathrm{Sc}}=(f, v)_{\mathrm{Sc}}
$$

for every $v \in W_{0}^{1, p(x)}\left(\Omega, C \ell_{n}^{1}\right) \cap$ ker div.

In the following we would like to point out the equivalence of solutions and weak solutions for the generalized Navier-Stokes equations; this point is similar to the case of the Stokes equations.

Theorem 5.2 Let $u \in W_{0}^{1, p(x)}\left(\Omega, C \ell_{n}^{1}\right)$ and $\pi \in L^{p(x)}(\Omega)$ be a solution of system (5.6)-(5.7). Then $u$ is a weak solution of system (5.1)-(5.3). If $u$ is a weak solution of the system (5.6)(5.7), then there exists a function $\pi \in L^{p(x)}(\Omega)$ such that the pair $(u, \pi)$ solves the system (5.1)-(5.3).

Proof The proof is quite similar to the proof of Theorem 4.2, so we omit it. We also refer the reader to a similar proof of Theorem 4.6.2 in [33] by replacing $Q$ with $Q_{a} B$.

Competing interests

The authors declare that they have no competing interests.

\section{Authors' contributions}

All authors carried out the theoretical studies, participated in the design of the study and drafted the manuscript. All authors read and approved the final manuscript.

\section{Author details}

${ }^{1}$ College of Power and Energy Engineering, Harbin Engineering University, Harbin, 150001, China. ${ }^{2}$ Department of Mathematics, Heilongjiang Institute of Technology, Harbin, 150050, China.

\section{Acknowledgements}

The authors would like to thank the referee for constructive suggestion which helps us to improve the quality of the paper. B Zhang was supported by the Research Foundation of Heilongjiang Educational Committee (Grant No. 12541667). 


\section{References}

1. Acerbi, E, Mingione, G: Regularity results for stationary electro-rheological fluids. Arch. Ration. Mech. Anal. 164, 213-259 (2002)

2. Sprößig, W: Fluid flow equations with variable viscosity in quaternionic setting. Adv. Appl. Clifford Algebras 17, 259-272 (2007)

3. Galdi, GP: An Introduction to the Mathematical Theory of the Navier-Stokes Equations. Springer, New York (2011)

4. Rajagopal, KR, Růžička, M: On the modeling of electrorheological materials. Mech. Res. Commun. 23, $401-407$ (1996)

5. Růžička, M: Electrorheological Fluids: Modeling and Mathematical Theory. Springer, Berlin (2000)

6. Gilbert, J, Murray, MAM: Clifford Algebra and Dirac Operators in Harmonic Analysis. Oxford University Press, Oxford (1993)

7. Delanghe, R, Sommen, F, Soucek, V: Clifford Algebra and Spinor-Valued Function. Kluwer Academic, Dordrecht (1992)

8. Doran, C, Lasenby, A: Geometric Algebra for Physicists. Cambridge University Press, Cambridge (2003)

9. Diening, L, Harjulehto, P, Hästö, P, Růžička, M: Lebesgue and Sobolev Spaces with Variable Exponents. Springer, Berlin (2011)

10. Fu, YQ: Weak solution for obstacle problem with variable growth. Nonlinear Anal. 59, 371-383 (2004)

11. Fu, YQ: The principle of concentration compactness in spaces $L^{p(x)}(\Omega)$ and its application. Nonlinear Anal. 71, 1876-1892 (2009)

12. Rădulescu, V, Zhang, BL: Morse theory and local linking for a nonlinear degenerate problem arising in the theory of electrorheological fluids. Nonlinear Anal., Real World Appl. 17, 311-321 (2014)

13. Zhikov, WV: Averaging of functionals of the calculus variations and elasticity theory. Izv. Akad. Nauk SSSR, Ser. Mat. 877, 675-710 (1986)

14. Zhikov, W: Solvability of the three-dimensional thermistor problem. Tr. Mat. Inst. Steklova 261, 101-114 (2008)

15. Kováčik, O, Rákosník, J: On spaces $L^{p(x)}$ and $W^{m, p(x)}$. Czechoslov. Math. J. 41, 592-618 (1991)

16. Edmunds, DE, Rákosník, J: Sobolev embedding with variable exponent. Stud. Math. 143, 267-293 (2000)

17. Edmunds, DE, Rákosník, J: Sobolev embedding with variable exponent II. Math. Nachr. 246, 53-67 (2002)

18. Fan, $X L$, Zhao, D: On the spaces $L^{p(x)}$ and $W^{m, p(x)}$. J. Math. Anal. Appl. 263, 424-446 (2001)

19. Fan, XL, Shen, JS, Zhao, D: Sobolev embedding theorems for spaces $W^{k, p(x)}(\Omega)$. J. Math. Anal. Appl. 262, 749-760 (2001)

20. Harjulehto, P, Hästö, P, Lê, UV, Nuortio, M: Overview of differential equations with non-standard growth. Nonlinear Anal. 72, 4551-4574 (2010)

21. Nolder, CA: A-Harmonic equations and the Dirac operator. J. Inequal. Appl. 2010, Article ID 124018 (2010)

22. Nolder, CA: Nonlinear A-Dirac equations. Adv. Appl. Clifford Algebras 21, 429-440 (2011)

23. Fu, YQ, Zhang, BL: Clifford valued weighted variable exponent spaces with an application to obstacle problems. Adv. Appl. Clifford Algebras 23, 363-376 (2013)

24. Fu, YQ, Zhang, BL: Weak solutions for elliptic systems with variable growth in Clifford analysis. Czechoslov. Math. J. 63 , 643-670 (2013)

25. Zhang, BL, Fu, YQ: Weak solutions for A-Dirac equations with variable growth in Clifford analysis. Electron. J. Differ. Equ. 2012, 227 (2012)

26. Lu, Y, Bao, G: The existence of weak solutions to non-homogeneous A-Dirac equations with Dirichlet boundary data. Adv. Appl. Clifford Algebras 24, 151-162 (2014)

27. Fu, YQ, Rădulescu, V, Zhang, BL: Hodge decomposition of variable exponent spaces of Clifford-valued functions and applications to Dirac and Stokes equations. Preprint

28. Zhang, BL, Fu, YQ, Rădulescu, V: The stationary Navier-Stokes equations in variable exponent spaces of Clifford-valued functions. Adv. Appl. Clifford Algebras 24, 231-252 (2014)

29. Molica Bisci, G, Rădulescu, V, Zhang, BL: Existence of stationary states for A-Dirac equations with variable growth. Adv. Appl. Clifford Algebras (2014). doi:10.1007/s00006-014-0512-y

30. Binlin, Z, Ferrara, M, Molica Bisci, G: An overview for nonlinear A-Dirac equations with variable growth. BSG Proc. 21, 14-26 (2014)

31. Diening, L, Málek, J, Steinhauer, M: On Lipschitz truncations of Sobolev function (with variable exponent) and their selected applications. ESAIM Control Optim. Calc. Var. 14, 211-232 (2008)

32. Gürlebeck, K, Sprößig, W: Quaternionic and Clifford Calculus for Physicists and Engineers. Wiley, New York (1997)

33. Gürlebeck, K, Sprößig, W: Quaternionic Analysis and Elliptic Boundary Value Problems. Birkhäuser, Boston (1990)

34. Ablamowicz, R (ed.): Clifford Algebras and Their Applications in Mathematical Physics. Volume 1: Algebra and Physics. Birkhäuser, Boston (2000)

35. Ryan, J, Sprößig, W (eds.): Clifford Algebras and Their Applications in Mathematical Physics. Volume 2: Clifford Analysis. Birkhäuser, Boston (2000)

36. Diening, L, Lengeler, D, Růžička, M: The Stokes and Poisson problem in variable exponent spaces. Complex Var. Elliptic Equ. 56, 789-811 (2011)

37. Kähler, U: On a direct decomposition in the space $L_{p}(\Omega)$. Z. Anal. Anwend. 4, 839-848 (1999)

38. Cerejeiras,, , Kähler, U: Elliptic boundary value problems of fluid dynamics over unbounded domains. Math. Methods Appl. Sci. 23, 81-101 (2000)

39. Finn, R: On the exterior stationary problem for the Navier-Stokes equations and associated perturbation problems. Arch. Ration. Mech. Anal. 19, 363-406 (1965) 